\title{
Epidemiologia das polidactilias: um estudo de casos e controles na população de Pelotas-RS
}

\author{
Epidemiology of polydactylies: a case-control study in the population of Pelotas-RS
}

\author{
Marcelo Boeing ${ }^{1}$, Luciene de Cassia F. Paiva ${ }^{2}$, Gilberto de Lima Garcias ${ }^{3,5}$, \\ Maria da Graça Martino Roth ${ }^{3}$, Iná S. Santos ${ }^{4}$
}

\section{Resumo}

Objetivo: o alvo deste estudo caso-controle de base hospitalar foi identificar e comparar casos de polidactilia isolada com recémnascidos normais, considerando as características familiares e maternas.

Métodos: os dados foram coletados através de entrevista com mães de casos e controles, no período pós-parto, de 1990 a 1998. Os casos foram definidos como recém-nascidos apresentando um dígito extra ou bífido nas mãos e/ou pés. Os controles foram os quatro recém-nascidos normais que nasceram após o caso, no mesmo hospital. Foram obtidas informações sobre o tipo de polidactilia, sexo e peso do recém-nascido, gemelaridade, consanguinidade parental, etnicidade, entre outras.

Resultados: a ancestralidade africana e a história familiar positiva de malformação congênita, especialmente de polidactilia, foram significantemente associados com a ocorrência desse tipo de anomalia congênita (OR bruto de 3,3; 10,0; e 55,0; respectivamente).

Conclusões: na população estudada a polidactilia isolada é uma das malformações mais freqüentes. Os achados de associação com etnicidade negro-africana confirmam dados da literatura. Estudos nesta mesma população, com um número maior de portadores desta malformação, poderiam melhorar o poder de associação e justificar os resultados apresentados.

J Pediatr (Rio J) 2001; 77 (2): 148-52: polidactilia, defeitos congênitos, negros/genética.

\section{Introdução}

Nas populações humanas em geral, muitos são os casos de malformações congênitas, cada qual apresentando características próprias quanto a incidência, influência genética, gravidade e morbidade associada. A polidactilia, uma

1. Faculdade de Medicina da Universidade Federal de Pelotas (UFPel)- RS.

2. Faculdade de Enfermagem e Obstetrícia da UFPel.

3. Departamento de Zoologia e Genética, Instituto de Biologia, UFPel.

4. Departamento de Medicina Social, Faculdade de Medicina, UFPel.

5. Escola de Medicina - UFPel.

\begin{abstract}
Objective: to identify and compare cases of isolated polydactyly with healthy newborns regarding familial and maternal characteristics.

Methods: we conducted this hospital-based case-control study from 1990 to 1998 . We collected data in interviews with mothers of cases and controls during the post-delivery period. Cases were defined as newborns presenting an extra or a bifid digit in hands and/ or feet. Controls were the four healthy newborns that were born after the case, at the same hospital. Information was gathered on type of polydactyly, gender and birthweight, twin pregnancy, parental consanguinity, ethnicity.

Results: African ancestry and positive family history of congenital malformation, especially polydactyly, were significantly associated with the occurrence of this type of congenital anomaly (crude OR of 3.3; 10.0; and 55.0, respectively).

Conclusion: isolated polydactyly was one of the most frequent malformations found in the studied population. The findings associated with black African ancestry confirm the data presented in the literature. Studies conducted on this same population, with a larger population of patients with this type of malformation, could investigate this association better and justify the results presented here.
\end{abstract}

J Pediatr (Rio J) 2001; 77 (2): 148-52: polydactyly, birth defects, blacks/genetics.

das malformações mais comumente encontradas, é caracterizada, clinicamente, pela manifestação de um dígito extra nas mãos e/ou pés, quando há divisão radial excessiva, ou um dígito largo ou bífido quando a divisão é incompleta ${ }^{1}$. Essas anomalias são classificadas em polidactilias pósaxiais ou pré-axiais, segundo seu aparecimento na face ulnar/fibular ou radial/tibial da mão ou pé, respectivamente. Podem ocorrer em ambos os membros e em ambos os lados do corpo concomitantemente ${ }^{2}$. 
A hereditariedade desta malformação, sendo seu fator causal, se comporta diferentemente conforme variações geográficas e conseqüentemente raciais, refletindo então diferentes manifestações na ocorrência de dígitos extras ${ }^{1}$. Na cidade de Pelotas (RS- Brasil), a polidactilia isolada é a segunda anomalia congênita mais freqüente ${ }^{3}$, com uma taxa de 15,6 casos a cada 10.000 nascimentos. O presente trabalho tem por objetivo traçar um perfil do comportamento desta malformação isolada (não associada a síndromes), na população de Pelotas.

\section{Material e Método}

O estudo foi conduzido nos 5 hospitais-maternidades de Pelotas, uma cidade com aproximadamente 300.000 habitantes ${ }^{4}$, localizada no sul do Brasil, num período de 9 anos (1990 a 1998). Os dados foram colhidos através da aplicação do questionário do ECLAMC (Estudo Colaborativo Latino-Americano de Malformações Congênitas). O questionário foi aplicado no hospital, logo após o parto, a todas as mães de crianças nascidas com polidactilia não associada a outra malformação (casos). Foram selecionadas como controles quatro crianças normais e nascidas nos mesmos hospitais após o nascimento de algum malformado com qualquer tipo de malformação. Setenta e cinco por cento dos controles eram emparelhados aos casos das demais malformações de acordo com o sexo. As mães dos controles foram entrevistadas com o mesmo questionário.

Foram estudadas as seguintes variáveis: tipos de polidactilia, sexo e peso do recém-nascido, idade materna, gemelaridade, consangüinidade parental, número de gestações, grau de instrução materna (escolaridade), etnia dos antepassados e história de malformados na família.

Os tipos de polidactilia foram agrupados em pós e préaxial, subdivididas segundo a localização em mão, pé ou ambos.

$\mathrm{O}$ sexo e o peso do recém-nascido foram obtidos do registro da maternidade. A idade da mãe foi obtida em anos. Gemelaridade referia-se à presença de mais de um feto como resultado da atual gestação. Consagüinidade parental foi definida como a união de parentes, em qualquer grau, na família.

A etnicidade foi considerada, a partir do reconhecimento pela mãe, do grupo ou grupos étnicos entre os ancestrais do recém-nascido, sendo estes: europeus latinos, europeus não-latinos, judeus, nativos, turcos, negros, orientais e outros. Essas etnias foram posteriormente agrupadas, segundo a cor, em brancos, negros e pardos, sendo classificados como brancos aqueles indivíduos cuja etnia negra fosse totalmente descartada. Pardos foram considerados aqueles que declararam algum tipo de miscigenação da raça negra com qualquer uma das demais. Mesmo reconhecendo que a questão de auto-conceito de cor e etnia é um fator de determinação incerta, optamos por este tipo de abordagem, uma vez que é o mesmo utilizado pelo Estudo Colaborativo Latino-Americano de Malformações Congênitas.
Investigou-se malformados na família como sendo a ocorrência de antepassados com anormalidades congênitas de qualquer tipo, segundo o conhecimento dos pais, não levando em consideração o grau de parentesco com o recém-nato.

Os dados referentes à população de Pelotas foram obtidos no IBGE (Instituto Brasileiro de Geografia e Estatística), segundo Censo realizado em 1991, atualizado em 1996.

A amostra foi obtida a partir de fichas de malformados que participam de um grande estudo latino-americano (Estudo Colaborativo Latino-Americano de Malformações Congênitas - ECLAMC), o qual foi aprovado pelas comissões científica e de ética de todos os hospitais de Pelotas e que dispensa o termo de consentimento, pois se trata de um estudo de base populacional.

Com um total de 81 casos e quatro controles para cada caso, o estudo teve um poder de $75 \%$ de detectar um risco relativo de 3,0 ou mais, significativo ao nível de 5\%.

\section{Resultados}

Durante os nove anos de coleta de dados houve em Pelotas aproximadamente 51.900 nascimentos nos hospitais-maternidades ${ }^{5}$. Neste período, ocorreram 81 casos de crianças polidáctilas, o que determinou uma prevalência de 15,6 casos para cada 10.000 nascimentos.

A Tabela 1 evidencia claramente a alta prevalência das polidactilias pós-axiais, que representam $73 \%$ dos casos especificados ocorridos em Pelotas. Fica evidente também a predominância da localização da mão como sede do dedo extra $(82,5 \%)$, independente do tipo de polidactilia.

Das crianças nascidas com polidactilia a maioria foi do sexo masculino $(65,4 \%)$, sendo a razão masculino:feminino de 1,9:1; no entanto, estas diferenças não são estatisticamente significativas (Tabela 2).

Tabela 1 - Tipo e localização das polidactilias, Pelotas, 1999

\begin{tabular}{lcc}
\hline Tipo de polidactilia & $\mathbf{N}^{\circ}$ de casos & $\%$ \\
\hline Pós-axial & & \\
$\quad$ Mão & 45 & 60,8 \\
$\quad$ Pé & 4 & 5,4 \\
$\quad$ Mão e Pé & 5 & 6,8 \\
Pré-axial & & \\
$\quad$ Mão & 5 & 6,8 \\
$\quad$ Pé & 2 & 2,7 \\
Não especificada & & \\
$\quad$ Mão & 11 & 14,9 \\
$\quad$ Pé & 1 & 1,3 \\
$\quad$ Mão e pé & 1 & 1,3 \\
\hline Total & $\mathbf{7 4}$ & $\mathbf{1 0 0}$ \\
\hline
\end{tabular}


Tabela 2 - Distribuição e efeitos brutos de características maternas e do recém-nascido sobre a ocorrência de polidactilia, de acordo com o status caso e controle, Pelotas, 1999

\begin{tabular}{|c|c|c|c|c|}
\hline Característica & $\operatorname{Casos}(n=81)^{1}$ & Controles $(n=324)^{1}$ & ORB (IC95\%) & $\mathbf{p}$ \\
\hline \multicolumn{5}{|l|}{ Sexo do recém-nascido } \\
\hline Masculino & $53(65,4 \%)$ & $185(57,8 \%)$ & $1,38(0,81-2,37)$ & 0,27 \\
\hline Feminino & $28(34,6 \%)$ & $135(42,2 \%)$ & 1,00 & \\
\hline \multicolumn{5}{|l|}{ Gemelaridade } \\
\hline Sim & $2(2,4 \%)$ & $5(1,5 \%)$ & $1,62(0,21-9,60)$ & 0,64 \\
\hline Não & $79(97,6 \%)$ & $319(98,5 \%)$ & 1,00 & \\
\hline \multicolumn{5}{|l|}{ Consangüinidade parental } \\
\hline Sim & $4(6,1 \%)$ & $12(3,7 \%)$ & $1,92(0,57-6,16)$ & 0,22 \\
\hline Não & $62(76,5 \%)$ & $286(88,2 \%)$ & 1,00 & \\
\hline \multicolumn{5}{|l|}{ Idade materna } \\
\hline$<20$ & $14(17,2 \%)$ & $52(16,0 \%)$ & 1,00 & 0,80 \\
\hline $20-25$ & $26(32,0 \%)$ & $114(35,1 \%)$ & $0,85(0,39-1,87)$ & \\
\hline $26-31$ & $21(25,9 \%)$ & $67(20,6 \%)$ & $1,16(0,51-2,69)$ & \\
\hline$>31$ & $17(20,9 \%)$ & $80(24,6 \%)$ & $0,79(0,33-1,87)$ & \\
\hline \multicolumn{5}{|l|}{ Número de gestações } \\
\hline 1 & $27(33,3 \%)$ & $113(34,8 \%)$ & 1,00 & $0,39^{2}$ \\
\hline 2 & $19(23,4 \%)$ & $84(25,9 \%)$ & $0,95(0,47-1,90)$ & \\
\hline 3 & $15(18,5 \%)$ & $51(15,7 \%)$ & $1,23(0,57-2,65)$ & \\
\hline$>3$ & $19(23,3 \%)$ & $63(19,2 \%)$ & $1,26(0,62-2,57)$ & \\
\hline \multicolumn{5}{|l|}{ Grau de instrução materna } \\
\hline Baixo & $57(70,3 \%)$ & $228(70,3 \%)$ & 1,00 & $0,39^{2}$ \\
\hline Médio & $14(17,2 \%)$ & $65(20,0 \%)$ & $0,86(0,43-1,71)$ & \\
\hline Alto & $3(3,7 \%)$ & $20(6,1 \%)$ & $0,60(0,14-2,23)$ & \\
\hline
\end{tabular}

1. Diferença em totais das variáveis devido a perda de informações. ORB (IC95\%): Razão de odds bruta e intervalo de confiança de $95 \%$ 2. p para tendência linear

As variáveis peso ao nascer, idade materna, gemelaridade, consangüinidade parental, número de gestações e grau de instrução materna (Tabela 2) não apresentaram diferença significativa entre casos e controles.

O peso médio ao nascer de casos e controles foi $3.195 \mathrm{~g}$ e $3.210 \mathrm{~g}$, respectivamente. A idade materna média de ambos os grupos foi 25 anos. Ocorreu um nascimento de gêmeos univitelinos entre os casos, sendo que ambas as crianças apresentaram as mesmas características quanto à polidactilia (pós-axial da mão). Cinco crianças que também tiveram irmãos gêmeos estavam entre os controles. A consangüinidade parental foi relatada por $6,1 \%$ dos casos e $3,7 \%$ dos controles.

As mães de casos e controles eram muito semelhantes quanto ao número médio de gestações e quanto à escolaridade. Em ambos os grupos as mães relataram uma média de duas gestações, incluindo a gestação atual. Embora 8,6\% dos casos fossem recém-nascidos de mães com mais de seis gestações, contra apenas $2,7 \%$ entre os controles, a diferença de paridade não foi estatisticamente significativa entre os dois grupos.

A Tabela 3 evidencia claramente as diferenças entre casos e controles no que se refere à cor de seus antepassados. Quando os grupos de estudo foram analisados somando-se os antepassados negros e pardos em relação a ante- passados brancos, observou-se que as crianças que possuíam ao menos um antepassado negro, representavam 20,3\% dos controles e $45,8 \%$ dos casos. Ter pelo menos um antepassado negro ou pardo associou-se a um odds bruto 3,31 vezes maior de ocorrência de polidactilia $(\mathrm{ORB}=3,31$; IC 95\% 1,86-5,91; $\mathrm{p}<0,001)$.

A variável "presença de malformado na família" (tabela 4) foi a que apresentou maior diferença entre os grupos de casos e controles. Entre os casos, 55\% relataram ao menos um parente com alguma malformação. $\mathrm{O}$ mesmo foi relatado por apenas $13 \%$ dos controles $(\mathrm{ORB}=10,18$; IC $95 \%$ $5,53-18,84 ; \mathrm{p}<0,001)$. Ao se investigar qual anormalidade, $81,3 \%$ dos casos relataram polidactilia, enquanto somente $7,2 \%$ dos antepassados dos controles que apresentavam alguma malformação eram polidáctilos.

Tabela 3 - Distribuição dos antepassados de casos e controles segundo a cor, Pelotas, 1999

\begin{tabular}{lcccr}
\hline Cor dos antepassados & Casos & \% & Controles & \% \\
\hline Negro & 16 & 22,2 & 33 & 11,2 \\
Pardo & 17 & 23,6 & 27 & 9,1 \\
Branco & 39 & 54,2 & 235 & 79,7 \\
\hline Total & $\mathbf{7 2}$ & $\mathbf{1 0 0}$ & $\mathbf{2 9 5}$ & $\mathbf{1 0 0}$ \\
\hline
\end{tabular}


Tabela 4 - Presença de malformados na família conforme status de caso ou controle, Pelotas, 1999

\begin{tabular}{lcccc}
\hline Malformados na família & Casos & $\boldsymbol{\%}$ & Controles & \% \\
\hline Sim & 45 & 55 & 42 & 13 \\
Não & 28 & 35 & 266 & 82 \\
Não souberam informar & 8 & 10 & 16 & 5 \\
\hline Total & $\mathbf{8 1}$ & $\mathbf{1 0 0}$ & $\mathbf{3 2 4}$ & $\mathbf{1 0 0}$ \\
\hline
\end{tabular}

ORB (IC95\%): 10,18(5,53-18,84)

$\mathrm{P}<0,001$

\section{Discussão}

Em populações urbanas altamente miscigenadas, diferenças entre países nas taxas de prevalência ao nascer de anomalias congênitas refletem amplamente as diferenças étnicas ${ }^{6}$. Na América do Sul, a prevalência de defeitos ao nascimento é observada segundo variações geográficas, principalmente devido a diferentes proporções na "mistura" tri-híbrida básica entre ameríndios, europeus latinos e negros africanos 7 .

Pelotas tem suas prevalências de anomalias congênitas influenciadas principalmente pelas etnias de europeus latinos e negros africanos. De acordo com o censo realizado em 1991, atualizado em 1996, a população local é formada por $85,5 \%$ de indivíduos de cor branca, 7,43\% de indivíduos de cor negra e 7,2\% de cor parda (mulatos, mestiços, caboclos, mamelucos e cafuzos). Os restantes $0,32 \%$ são distribuidos entre indivíduos de cor amarela ou sem declaração ou que não sabiam informar a própria cor ${ }^{4}$.

Nesta localidade, a polidactilia isolada é uma das malformações mais frequientes, perdendo em prevalência ao nascimento somente para pé torto congênito ${ }^{3}$.

A taxa geral de 15,6 casos a cada 10.000 nascimentos, obtida nos 9 anos de estudo, é formada predominantemente pela polidactilia pós-axial. Como $20,9 \%$ dos casos não foram especificados quanto ao tipo de polidactilia ocorrida, acredita-se que a prevalência das pós-axiais, em especial a que ocorre na mão, seja um pouco maior. Estima-se, portanto, que as taxas de polidactilia girem em torno de 1,7/10.000 para pré-axial e 13,9/10.000 para pós-axial.

A polidactilia pré-axial pode encontrar-se mais associada à etnia chinesa e a índios americanos, sendo na maioria das vezes unilateral, não familiar e envolvendo principalmente membros superiores. É pequeno o número de habitantes descendentes de chineses e de índios em Pelotas, o que justifica sua baixa prevalência. Esse tipo de polidactilia é relatado como tendo comportamento autossômico recessivo e é freqüentemente um componente de muitas síndromes, tendo suas características genéticas vinculadas a estas, mudando então para autossômica dominante, dependendo da síndrome em questão ${ }^{1}$.
Em relação à polidactilia pós-axial, quase a totalidade dos casos ocorridos, autores como McKusick (1990) e também Frazier (1960) relataram uma freqüência 10 vezes maior em negros do que em brancos. Já Scott-Emuakpor e Madueke (1976) relataram uma prevalência total de 225/ 10.000 na Nigéria, enquanto Castilla et al. (1997) relataram uma prevalência de 14,3/10.000 na América Latina, taxa essa que se aproxima muito da encontrada em Pelotas. Tenconi et al. (1990) relataram uma prevalência de 4,8/ 10.000 na Itália e Handforth (1950) registrou apenas um caso entre 5842 chineses prisioneiros em Hong Kong. Neste mesmo estudo foram registrados 13 prisioneiros com polidactilia pré-axial.

Polidactilia pós-axial em humanos, em geral, é considerada uma característica autossômica dominante, com penetrância baixa e expressividade variável ${ }^{14}$, tendo sido também relatada como uma característica autossômica recessiva $^{15-17}$ e em associação com várias síndromes ${ }^{18}$. Orioli (1995) sugeriu uma distorção de segregação familiar para polidactilia pós-axial devido à ação de um gene modificado-recessivo ligado ao sexo, mais freqüente em negros, principalmente quando relacionado à polidactilia pós-axial da mão. Este comportamento genético se expressa de maneira clara na população de Pelotas afetada por esta malformação, visto a alta frequiência de polidactilia pós-axial (especialmente a pós-axial da mão) e a associação de crianças polidáctilas com ancestrais negros, minoria dos habitantes locais, além do grande número de casos de parentes também polidáctilos.

Este estudo não detectou diferença significativa quanto ao sexo, o que pode ser explicado pela super-representatividade de meninos entre os controles, decorrente do emparelhamento desses controles quanto ao sexo, com recémnascidos com outras malformações. Ainda assim, a proporção foi de 1,9:1 de masculino/feminino. Outros autores relataram uma maior freqüência de casos desta malformação no sexo masculino. Nos trabalhos citados anteriormente, Scott-Emuakpor e Madueke (1976), na Nigéria, relataram 1,5:1 de masculino/feminino, o mesmo que Tenconi et al. (1990) na Itália. Na América do Sul, Castilla et al. (1973) relataram 1,3:1 para masculino/feminino em relação a pósaxial e 2,9:1 para pré-axial.

Em relação à polidactilia pós-axial da mão, Castilla et al. (1997), ao analisarem 1.733 casos ocorridos na América Latina, num período de 26 anos, relataram associação com etnicidade negro-africana, sexo masculino, gemelares, baixa educação materna e consangüinidade parental, sendo também freqüente a recorrência familiar. O atual estudo não detectou associação significativa entre essas características e polidactilia. Ressalta-se, no entanto, que o tamanho da amostra estudada teve um poder de apenas $75 \%$ para detectar associações (odds ratios) iguais ou superiores a 3,0 .

Nesse estudo, a ocorrência de polidactilia associou-se positivamente à existência de ancestrais da raça negra e de familiares com história de malformações congênitas. A 
ascendência negra representou um odds 3 vezes maior que o observado entre crianças de famílias brancas. A característica mais fortemente associada com a presença de polidactilia, no entanto, foi a história positiva de ocorrência de malformações em outras pessoas da família, sendo o odds 10 vezes maior do que o observado nos demais. A própria polidactilia foi a malformação mais freqüentemente relatada $(81,3 \%)$ entre os familiares dos casos. Ter um familiar com história de polidactilia neste estudo implicou em um odds 55 vezes maior de o recém-nascido apresentar a mesma malformação (ORB=55,00; IC 95\% 26,02-118, 25; $\mathrm{p}<0,001)$. Parte do efeito da história familiar, particularmente da história de polidactilia, deve ser decorrente de viés de memória ${ }^{20}$. Mães de recém-nascidos malformados lembram com maior frequiência de fatos ou outras malformações ocorridas no passado do que mães de recémnascidos normais. O recordatório materno também pode ter melhorado o conjunto total de informações (raça, antecedentes familiares, etc.), que foram utilizados para as comparações.

\section{Referências bibliográficas}

1. Stevenson RE, Hall J, Goodman RM. The Polydactylies. In: Stevenson RE, Hall J, Goodman RM. Human malformations and related anomalies. New York: Oxford University Press; 1993. p 818-28.

2. Temtamy SA, McKusick VA. The genetics of hand malformations. BDOAS XIV 1978 (3):376,379,391,405.

3. Dutra MG. XXVII Reunion Anual del Estudio Colaborativo Latino Americano de Malformações Congênitas - Uruguai 1995. Documento final. Rio de Janeiro; 1996.

4. IBGE - Instituto Brasileiro de Geografia e Estatística; Censo de 1991.

5. Garcias GL, Roth MG, Crespo CC, Sauressig S. Congenital defects monitoring program in the city of Pelotas, $\mathrm{RS}-\mathrm{Brasil}$. $\mathrm{Br}$ J Genet 1996; 2:188.

6. Lopez-Camelo JS, Cabello PH, Dutra MG. A simple model for the estimation of congenital malformation frequency in racially mixed populations. Braz J Genet 1996; 19:659-63.

7. Lopez-Camelo JS, Orioli IN. Heterogeneous rates for birth defects in Latin America: hints on causality. Genet Epidemiol 1996; 13:451-68.

8. McKusick VA. Mendelian inheritance in man. Baltimore: Johns Hopkins University Press; 1990.
9. Frazier TM. A note on race-specific congenital malformation rates. Am J Obstet Gynecol 1960; 80:184-5.

10. Scott-Emuakpor B, Madueke EDN. The study of genetic variation in Nigeria II. The genetics of polydactyly. Hum Hered 1976; 26:198.

11. Castilla EE, Dutra MG, Fonseca RL, Paz JE. Hand and foot postaxial polydactyly: Two differents traits. Am J Med Genet 1997; 73:48-54.

12. Tenconi R, Clementi M, Turolla L, et al. Polydactyly: an epidemiological and genetic study based on 315,015 consecutive newborns in North-East Italy in the period 1981-1988. Proceedings Greenwood Genetics Center 1990; 9:83.

13. Handforth JR. Polydactylism of the hand in Southern Chinese. Anat Rec 1950; 106:119.

14. Castilla E, Paz J, Mutchinik O, Munoz E, Giorgutti E, Gelman Z. Polydactyly: a genetic study in South America. Am J Hum Genet $1973 ; 25: 405-12$.

15. Mohan J. Postaxial polydactyly in three Indian families. J Med Genet 1969; 6:196-200.

16. Cantu J-M, Del Castillo V, Cortes R, Vrrusti J. Autossomal recessive postaxial polydactyly: report of a family. Birth Defects 1974; 5:19-22.

17. Mollica F, Li Volti S, Sorge G. Autossomal recessive postaxial polydactyly type A in a Sicilian family. J Med Genet 1978; 15:212-16.

18. Merlob P, Grunebaum M, Reisner SH. A newborn infant with craniofacial dysmorfism and polysyndactyly. Acta Paediatr Scand 1981; 70:275-77.

19. Orioli IM. Segregation distortion in the offspring of AfroAmerica fathers with postaxial polydactyly. Am J Hum Genet 1995; 5:1207-11.

20. Schlesselman JJ. Case-control studies: design, conduct, analysis. New York: Oxford University Press; 1982.

Endereço para correspondência:

Dr. Marcelo Boeing

Rua Barão de Santa Tecla, 1180 - ap. 41

Pelotas - RS - CEP 96010-140

E-mail:1paiva@loja.net 